\title{
The results of a sociological survey on the quality of laboratory services of patients in Tashkent region
}

\section{Mukhamedjanova Nodira Ismaildjanovna}

Tashkent Institute of Postgraduate medical education. Senior lecturer at the Department of Clinical Laboratory Diagnostics of, Ph.D in Medicine

\section{Email address:}

araukariy77@mail.ru (Mukhamedjanova Nodira Ismaildjanovna)

\section{To cite this article:}

Mukhamedjanova Nodira Ismaildjanovna. The results of a sociological survey on the quality of laboratory services of patients in Tashkent region. Journal of research in health science. Vol. 1, No. 2, 2018, pp. 67-73. DOI $10.26739 / 2523-1243$

\section{dofं http://dx.doi.org/10.26739/2523-1243/-2018-1-2-11}

\begin{abstract}
: the most popular and popular among the treatment and diagnostic activities are laboratory studies. According to the WHO, the proportion of laboratory tests is $75-90 \%$ of the total number of different types of medical and diagnostic activities conducted by patients in medical institutions. Clinical medicine is in constant search for optimal diagnostic studies, and the qualification of the doctor directly affects the result of the examination of the patient. To meet the quality requirements of the international standardization of ISO 9000-9001 families, much remains to be done. At present, the development and scientific substantiation of measures to optimize their activities in all areas of Uzbekistan are needed. In this connection, the characterization of the current state and dynamics of the development of the diagnostic service in the case of the Tashkent region and the rationale for measures to improve it, is undoubtedly an urgent task. Studies of the organization of medical and diagnostic assistance to the population of the Tashkent region in the context of modernizing the health care system to justify measures to improve it was the main task of this study.
\end{abstract}

Keywords: quality control, questionnaires, laboratory diagnostics, questionnaires.

Under the system of quality management of medical care means a set of structural elements and functional mechanisms that ensure the achievement of a socially acceptable level of the main significant characteristics of interaction, patients with a medical subsystem. The process of improving the quality assurance system is connected with the study and development of its structural, procedural and effective components, controls, including standards, expert assessments, statistical indicators and the results of opinion polls (Minyaev VA et al., 2003).

The creation of a quality management system for clinical laboratory research, 
Mukhamedjanova Nodira Ismaildjanovna. The results of a sociological survey on the quality of laboratory services of patients in Tashkent region.

taking into account all its components and a variety of evaluation methods, is an urgent issue in modern laboratory medicine (Kishkun AA, 2006).

The purpose of this study was to study the level of patient satisfaction with the quality of laboratory services for medical and diagnostic assistance to the population of the Tashkent region in the context of healthcare modernization.

An analysis of the opinion of the population about the organization of the diagnostic process was carried out by means of a questionnaire. The survey involved patients from public health facilities $(n=490)$. Among the consumers of medical diagnostic services, persons aged $30-39$ years were $33.7 \%$, 20-29 years old $-2.4 \%$, in $40-49$ years $19.4 \%$, in 50 59 years $-15.6 \%$, at the age of 60 and over $-7.8 \%$. A higher activity of women was noted $(61.3 \%)$, the proportion of men was $38.7 \%$, pensioners were $22.8 \%$, disabled people $9.4 \%$.

The majority $(71.0 \%)$ of the patients asked for a laboratory examination in connection with the existing disease, $13.0 \%$ made an independent decision to perform such a survey, $9.4 \%$ of those who needed it needed the results of the tests for a preventive examination, $4.4 \%$ consultations for a pregnancy survey, and $2.2 \%$ required a certificate of health.

Primarily (in $78.3 \%$ of cases), the respondents were sent to a laboratory examination by treating physicians: district or family doctors $(25.4 \%)$, specialist doctors $(26.8 \%)$ and hospital doctors $(26.1 \%) .16 .6 \%$ came to the survey on their own initiative or on the advice of friends, which may indicate a lack of interaction between patients and treating physicians. At the same time, the latter.
The indicator is important in the formation of marketing strategy (especially promising are patients younger than 20 years).

It is important to note that in $55.1 \%$ of cases, patients fully $(29.0 \%)$ or partially $(26.1 \%)$ paid for laboratory tests on their own. The remaining half $(44.9 \%)$ underwent a laboratory examination free of charge. If at the age of 20 years only $14.2 \%$ of the respondents were surveyed free of charge, then in 20-39 years more than a third $(35.0 \%)$, over the age of 60 almost half $(45.9 \%)$, and in the age group of $50-59$ years old the majority $(72.7 \%)$ patients.

Respondents were asked to assess the quality of the diagnostic service. When asked about the degree of confidence in the results of diagnostic tests, only half of the respondents $(48.8 \% \pm 2.18)$ assess the "satisfactory" activity of the laboratory where they are being treated, a little more than a third $(19.1 \% \pm 2.08)-" 29.4 \%$ partially trusted, $2.7 \%$ - considered them unreliable.

$42.4 \%$ of patients indicated certain reasons for choosing a particular laboratory, each of which identified from one to four reasons. Almost a third (30.5\%) of the respondents were guided mainly by the recommendation of the attending physician, a quarter $(23.7 \%)$ for the availability of the necessary assortment of studies. For $20.3 \%$, the good reputation of the laboratory was significant, for $18.6 \%$, the speed of obtaining results and proximity to the place of residence. The question of the availability of prices when choosing a laboratory was of interest only to $8.5 \%$ of patients.

About two thirds of the respondents $(67.2 \%)$ learned about the rules of 
preparation for the analysis, the third (32.8\%) received such information from the laboratory staff. As one would expect, patients of commercial medical organizations more $(61.5 \%)$ turned to laboratory staff for information on preanalytical training. Despite the availability of such information, almost every tenth respondent $(8.6 \%)$ did not comply with the recommendations received.

Studying the level of patient satisfaction with certain aspects of the organization of a laboratory examination showed that the process of registering a referral to a laboratory study did not satisfy to one degree or another every fifth $(20.7 \%)$ of the patient (mostly people under the age of 20 and 50-59 years who were examined free of charge ).

One third of patients $(32.6 \%)$ passed tests without waiting in line. The rest were waiting for a turn for examination (including $2.9 \%$ more than half an hour). The majority of respondents $(85.0 \%)$ had the opportunity to sit, waiting for their turn. It is important to note that one third (30.7\%) of patients were in emotional discomfort, waiting for their turn, which could affect the results of analyzes, since only $50 \%$ of CSLs have a sample room.

In general, patients were significantly elevated (by 4.62 points in the five-point system) to assess the professionalism of the medical staff when taking blood for the study. The most excellent ratings $(92.9 \%)$ were given by respondents surveyed in commercial medical organizations.

It is important to note that one fifth (20.7\%) of respondents was not satisfied with the terms of the study and the results (to a greater extent, it is typical for those surveyed in public health facilities).
Half (52.9\%) of the respondents received the results of the tests from the attending physician, more than a third $(37.0 \%)$ of the patients themselves came for a response to the laboratory, the rest used the Internet (7.2\%) and telephone $(2.9 \%)$.

The majority $(85.0 \%)$ of patients evaluated the overall conditions for conducting laboratory tests as "excellent" and "good". Among the respondents surveyed in commercial medical organizations, the number of "excellent" assessments was higher $(71.4 \%)$ than in the group of patients examined in laboratories of state outpatient and outpatient clinics (47.5\%) and hospitals (45.5\%).

The survey showed that $32.4 \%$ of respondents had any problems with a laboratory examination. On average, respondents noted $1.46 \pm 0.13$ problems per person. It should be noted that those who applied to commercial medical organizations were more demanding on the level of service, and the average number of problems in private laboratories $(2.00 \pm 0.47)$ was higher compared to respondents surveyed in city hospitals $(1.00 \pm 0,00)$, polyclinics $(1.57 \pm 0.24)$ and clinics $(1.25 \pm 0.23)$. The most frequent problems were: longterm analysis (as indicated by $7.9 \%$ of respondents), territorial remoteness of the laboratory (6.4\%), availability of queues for analysis and discomfort in the room (5.7\%), difficulties in registration directions for laboratory research $(4.3 \%)$; Insufficient staff attentiveness $(3.6 \%)$. These problems influenced the attitude towards a particular laboratory.

Indirect evidence of the dissatisfaction (partial satisfaction) of 
Mukhamedjanova Nodira Ismaildjanovna. The results of a sociological survey on the quality of laboratory services of patients in Tashkent region.

patients with the organization and the quality of laboratory research is the fact that only slightly more than half (52.7\%) of respondents expressed an intention to again conduct research in the same laboratories (if necessary), and less than half $(45,6 \%)$ are going to recommend the laboratory, in which they passed the examination, to friends and acquaintances. Respondents of commercial medical organizations at a higher percentage $(64.3 \%)$ expressed their intention to recommend the laboratory to other users as compared to those surveyed in state outpatient clinics (48.2\%) and hospitals (40.0\%).

Thus, the results of the population survey indicate that the main problems in organizing a laboratory survey that reduce the level of satisfaction with its quality are the speed of obtaining the result and its questionable accuracy. Such a human factor as disrespectful attitude The results of a sociological survey on the quality of laboratory services of patients in Tashkent region Mukhamedzhanova N.I.

Under the system of quality management of medical care means a set of structural elements and functional mechanisms that ensure the achievement of a socially acceptable level of the main significant characteristics of interaction, patients with a medical subsystem. The process of improving the quality assurance system is connected with the study and development of its structural, procedural and effective components, controls, including standards, expert assessments, statistical indicators and the results of opinion polls (Minyaev VA et al., 2003).

The creation of a quality management system for clinical laboratory research, taking into account all its components and a variety of evaluation methods, is an urgent issue in modern laboratory medicine (Kishkun AA, 2006).

The purpose of this study was to study the level of patient satisfaction with the quality of laboratory services for medical and diagnostic assistance to the population of the Tashkent region in the context of healthcare modernization.

An analysis of the opinion of the population about the organization of the diagnostic process was carried out by means of a questionnaire. The survey involved patients from public health facilities $(n=490)$

Among the consumers of medical diagnostic services, persons aged 30-39 years were $33.7 \%$, 20-29 years old $-2.4 \%$, in $40-49$ years $19.4 \%$, in $50-59$ years $15.6 \%$, at the age of 60 and over $-7.8 \%$. A higher activity of women was noted $(61.3 \%)$, the proportion of men was $38.7 \%$, , pensioners were $22.8 \%$, disabled people $9.4 \%$.

The majority $(71.0 \%)$ of the patients asked for a laboratory examination in connection with the existing disease, $13.0 \%$ made an independent decision to perform such a survey, $9.4 \%$ of those who needed it needed the results of the tests for a preventive examination, $4.4 \%$ consultations for a pregnancy survey, and $2.2 \%$ required a certificate of health.

Primarily (in $78.3 \%$ of cases) the respondents were sent to a laboratory examination by treating physicians: district or family doctors (25.4\%), specialist doctors $(26.8 \%)$ and hospital doctors (26.1\%). $16.6 \%$ came to the survey on their own initiative or on the advice of friends, which may indicate a lack of interaction between patients and treating physicians. At the same time, the latter 

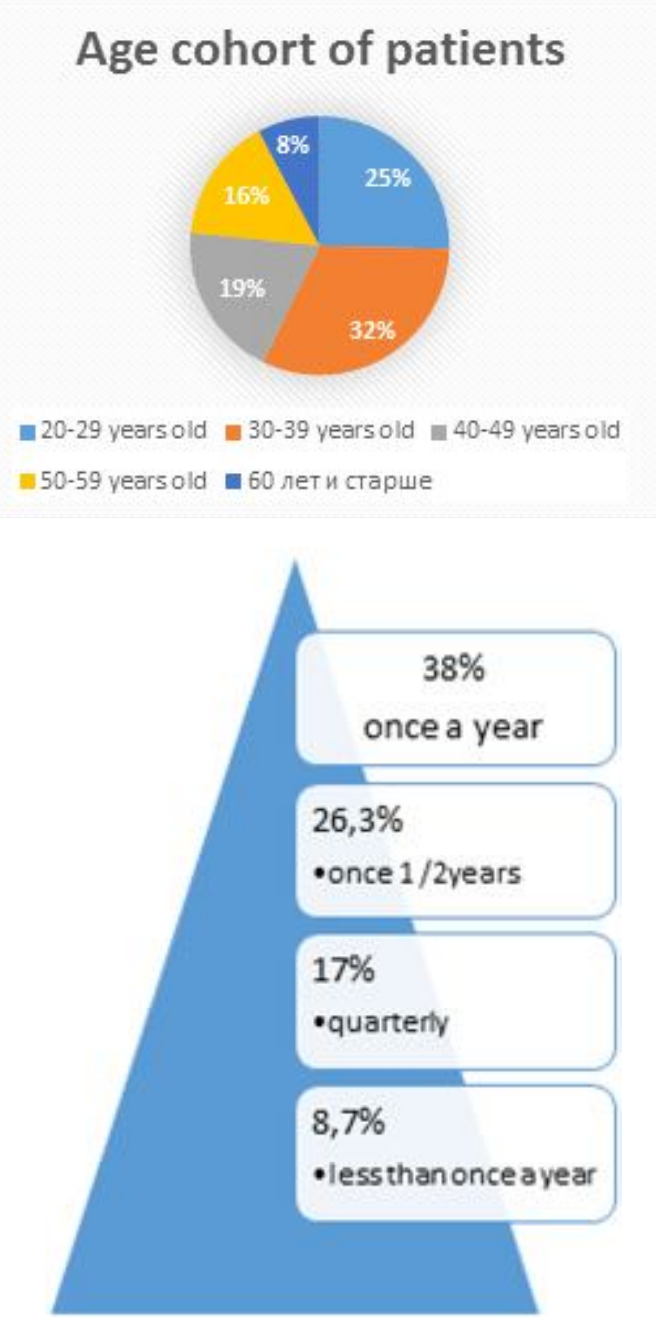

the indicator is important in the formation of marketing strategy (especially promising are patients younger than 20 years).

It is important to note that in $55.1 \%$ of cases, patients fully $(29.0 \%)$ or partially $(26.1 \%)$ paid for laboratory tests on their own. The remaining half $(44.9 \%)$ underwent a laboratory examination free of charge. If at the age of 20 years only $14.2 \%$ of the respondents were surveyed free of charge, then in 20-39 years more than a third $(35.0 \%)$, over the age of 60 almost half $(45.9 \%)$, and in the age group of 50- 59 years old the majority $(72.7 \%)$ patients.

Respondents were asked to assess the quality of the diagnostic service. When asked about the degree of confidence in the results of diagnostic tests, only half of the respondents $(48.8 \% \pm 2.18)$ assess the "satisfactory" activity of the laboratory where they are being treated, a little more than a third $(19.1 \% \pm 2.08)-" 29.4 \%$ partially trusted, $2.7 \%$ - considered them unreliable.

$42.4 \%$ of patients indicated certain reasons for choosing a particular laboratory, each of which identified from one to four reasons. Almost a third (30.5\%) of the respondents were guided mainly by the recommendation of the attending physician, a quarter $(23.7 \%)$ for the availability of the necessary assortment of studies. For $20.3 \%$, the good reputation of the laboratory was significant, for $18.6 \%$, the speed of obtaining results and proximity to the place of residence. The question of the availability of prices when choosing a laboratory was of interest only to $8.5 \%$ of patients.

About two thirds of the respondents $(67.2 \%)$ learned about the rules of preparation for the analysis, the third $(32.8 \%)$ received such information from the laboratory staff. As one would expect, patients of commercial medical organizations more $(61.5 \%)$ turned to laboratory staff for information on preanalytical training. Despite the availability of such information, almost every tenth respondent $(8.6 \%)$ did not comply with the recommendations received.

Studying the level of patient satisfaction with certain aspects of the organization of a laboratory examination showed that the process of registering a referral to a laboratory study did not satisfy to one degree or another every fifth (20.7\%) of the patient (mostly people under the 
Mukhamedjanova Nodira Ismaildjanovna. The results of a sociological survey on the quality of laboratory services of patients in Tashkent region.

age of 20 and 50-59 years who were examined free of charge).

One third of patients $(32.6 \%)$ passed tests without waiting in line. The rest were waiting for a turn for examination (including $2.9 \%$ more than half an hour). The majority of respondents $(85.0 \%)$ had the opportunity to sit, waiting for their turn. It is important to note that a third (30.7\%) of patients was in a state of emotional discomfort, waiting for its turn, which could affect the results of the analyzes, because only $50 \%$ of the KDL have a sample room.

In general, patients were significantly elevated (by 4.62 points in the five-point system) to assess the professionalism of the medical staff when taking blood for the study. The most excellent ratings $(92.9 \%)$ were given by respondents surveyed in commercial medical organizations.

It is important to note that one fifth (20.7\%) of respondents was not satisfied with the terms of the study and the results (to a greater extent, it is typical for those surveyed in public health facilities).

Half (52.9\%) of the respondents received the results of the tests from the attending physician, more than a third $(37.0 \%)$ of the patients themselves came for a response to the laboratory, the rest used the Internet (7.2\%) and telephone (2.9\%).

The majority $(85.0 \%)$ of patients evaluated the overall conditions for conducting laboratory tests as "excellent" and "good". Among the respondents surveyed in commercial medical organizations, the number of "excellent" assessments was higher (71.4\%) than in the group of patients examined in laboratories of state outpatient and outpatient clinics $(47.5 \%)$ and hospitals (45.5\%).
The survey showed that $32.4 \%$ of respondents had any problems with a laboratory examination. On average, respondents noted 1.46 ë 0.13 problems per person. It should be noted that those who applied to commercial medical organizations were more demanding on the level of service, and the average number of problems in private laboratories $(2.00 \pm 0.47)$ was higher compared to respondents surveyed in city hospitals $(1.00 \pm 0,00)$, polyclinics $(1.57$ $\pm 0.24)$ and clinics $(1.25 \pm 0.23)$. The most frequent problems were: long-term analysis (as indicated by $7.9 \%$ of respondents), territorial remoteness of the laboratory (6.4\%), availability of queues for analysis and discomfort in the room $(5.7 \%)$, difficulties in registration directions for laboratory research $(4.3 \%)$; Insufficient staff attentiveness (3.6\%). These problems influenced the attitude towards a particular laboratory.

Indirect evidence of the dissatisfaction (partial satisfaction) of patients with the organization and the quality of laboratory research is the fact that only slightly more than half $(52.7 \%)$ of respondents expressed an intention to again conduct research in the same laboratories (if necessary), and less than half $(45,6 \%)$ are going to recommend the laboratory, in which they passed the examination, to friends and acquaintances. Respondents of commercial medical organizations at a higher percentage $(64.3 \%)$ expressed their intention to recommend the laboratory to other users as compared to those surveyed in state outpatient clinics (48.2\%) and hospitals (40.0\%).

Thus, the results of the population survey indicate that the main problems in organizing a laboratory survey that reduce the level of satisfaction with its 
quality are the speed of obtaining the result and its questionable accuracy. Such a human factor as disrespectful attitude medical workers of CSL, queues for delivery of biological material and discomfort at its fence patients also consider significant. No less important is the range of laboratory tests performed and the qualifications of LDS doctors.

\section{References}

1. Horovskaya LA, Petrova NG, Vishnyakov NI, Emanuel VL, Ivanova LI, Kallner A. Optimization of the quality management system for laboratory research // Clinical laboratory diagnostics. - № 9. - 2006. - C. 7.

2. Horovskaya LA, Callner A., Petrova NG, Vishnyakov NI, Emanuel VL The main directions of improving the regional quality management system of the laboratory service // Human Ecology. Appendix 3. - 2006. - P. 136-137.

3. Moshkin AV, Dolgov VV Quality assurance in clinical laboratory diagnostics. M. 2004. -188p.

4. VVMenshikov. Standardization in laboratory medicine in Russia: first results and further plans. / / Problems of standardization in health care, I, 2009

5. National Committee for Clinical Laboratory Standards. Internal Quality Control: Principles and Definitions; C-24A. 